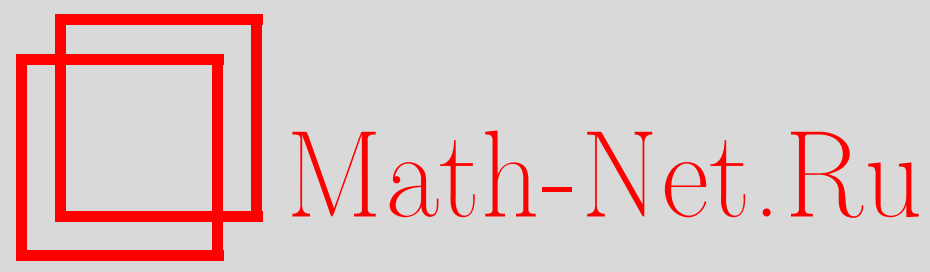

Р. И. Григорчук, Ветвящиеся группы, Матем. заметки, 2000, том 67, выпуск 6, 852-858

DOI: https://doi.org/10.4213/mzm903

Использование Общероссийского математического портала Math-Net.Ru подразумевает, что вы прочитали и согласны с пользовательским соглашением http://www.mathnet.ru/rus/agreement

Параметры загрузки:

IP : 3.93.64.190

26 апреля 2023 г., 15:18:26

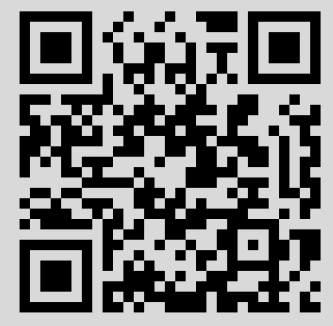


УДК 512.54

\title{
ВЕТВЯЩИЕСЯ ГРУППЫ
}

\section{Р.И. Григорчук}

\begin{abstract}
Определен класс ветвящихся групп (как в абстрактной, так и в проконечной категориях). Установлена связь этого класса с классом экстремальных групп. Исследованы свойства ветвящихся групп. Указаны приложения конгруэнц-свойства для теории проконечных ветвящихся групп. Доказана слабая максимальность параболических подгрупп в ветвящихся группах.

Библиографой: 18 названий.
\end{abstract}

В этой заметке мы определяем класс ветвящихся групп (как в абстрактной, так и проконечной категориях), формулируем основные утверждения о ветвящихся группах и обсуждаем роль этого класса в контексте фундаментальных проблем теории групп. При этом вводится ряд понятий, представляющих интерес с точки зрения теории групп, действующих на деревьях. Более подробное изложение дано в [1].

1. Группы, действующие на корневых деревьях. Пусть $\bar{m}=\left\{m_{n}\right\}_{n=0}^{\infty}-$ последовательность натуральных чисел, больших или равных 2 , назьваемая индексом ветвления, $T=T_{\bar{m}}-$ сферически однородное корневое дерево, определенное индексом $\bar{m}$ (т. е. корневая вершина имеет индекс ветвления $m_{0}$, каждая из вершин первого уровня имеет индекс ветвления $m_{1}$ и т. д.; при этом уровень вершины - это расстояние до корневой вершины).

Обозначим через Aut $T$ группу автоморфизмов дерева $T$ таких, что корневая вершина остается неподвижной, а вершины одного уровня переставляются, сохраняя при этом отношение инцидентности.

Группа Aut $T$ обладает естественной топологией, превращающей ее в проконечную группу, при этом Aut $T=\lim _{n}$ Aut $T_{[n]}$, где $T_{[n]}-$ поддерево в $T$, состоящее из вершин до $n$-го уровня включительно.

Граница дерева $T$ (т. е. множество геодезических путей, соединяющих корневую вершину с бесконечностью) обозначается $\partial T$. Группа Aut $T$ действует также и на гранище. Как отмечено в [1], при подходящем выборе метрики $\rho$ на границе пара $(\partial T, \rho)$ является универсальной моделью однородного ультраметрического пространства.

Со всякой подгруппой $G \leqslant$ Aut $T$ связаны следуюшие групшы:

$\operatorname{st}_{G}(u)=\left\{g \in G: u^{g}=u\right\}-$ стабилизатор вериины $u$, $\operatorname{st}_{G}(n)=\bigcap_{|u|=n} \operatorname{st}_{G}(u)-$ стабилизатор $n$-го уровня $(|u|$ обозначает уровень вершины $u$ ),

$\operatorname{rist}_{G}(u)=\left\{g \in G: v^{g}=v, v \in T \backslash T_{u}\right\}-$ жесткий стабилизатор вершины $и$ $\left(T_{u}\right.$ обозначает поддерево с корневой вершиной $\left.u\right)$, 
$\operatorname{rist}_{G}(n)=\left\langle\operatorname{rist}_{G}(u):|u|=n\right\rangle-$ группа, порожденная жесткими стабилизаторами вершин $n$-го уровня и назьваемая жсестким стабилизатором $n$-го уровня.

Так как жесткие стабилизаторы вершин одного уровня коммутируют, то rist $G(n)$ изоморфен прямому произведению жестких стабилизаторов вершин $n$-го уровня.

Стабилизаторы всегда имеют конечньй индекс в $G$, в то время как жесткий стабилизатор может быть тривиальным. Требование того, чтобы жесткие стабилизаторы уровней были подгруппами конечного индекса в $G$ (или хотя бы нетривиальньпи подгруппами), приводит к понятию ветвящейся (соответственно слабо ветвящейся) группы (см. ниже определение 1).

Действие группы $G$ на дерево $T$ называется сферически транзитивным. если оно транзитивно на каждом уровне дерева. В этом случае стабилизаторы (жесткие стабилизаторы) вершин одного уровня сопряжены между собой.

Пусть $e \in \partial T$ - элемент границы, обозначим $P=\operatorname{st}_{G}(e)-$ стабилизатор пути $e$. Подгруппа $P$ назьвается параболической.

2. Ветвящиеся группы. Здесь мы даем вариант определения ветвящейся групш, отличный от приведенного в [2].

ОПРЕДЕЛЕниЕ 1. а) Группа $G$ называется ветвящейся, если существует точное сферически транзитивное действие $G$ на некотором сферически однородном дереве $T_{\bar{m}}$ такое, что $\left|G: \operatorname{rist}_{G}(n)\right|<\infty, n=1,2, \ldots$.

б) Группа $G$ назьвается слабо ветвящ, йся, если при условиях предыдущего пункта определения $\operatorname{rist}_{G}(n) \neq 1, n=1,2, \ldots$.

в) Проконечная группа $G$ назьвается ветвящейся (слабо ветвящейся), если для некоторого дерева $T_{\bar{m}}$ сушествует вложение $G$ в Aut $T_{\bar{m}}$ (в категории проконечных групп), при котором вьполнено условие пункта а) (пункта б)).

Очевидно, всякая ветвящаяся группа является и слабо ветвящейся и что слабо ветвящаяся грушпа является бесконечной.

Мы намерены определить регулярно ветвящиеся группы. Дерево $T=T_{\bar{m}}$, для которого последовательность $\bar{m}$ является постоянной со значением $d \geqslant 2$, назьвается

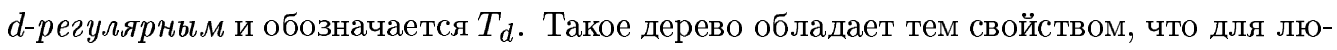
бой вершины $u$ поддерево $T_{u}$, растушее из этой вершины, изоморфно $T$. Таким образом, определено вложение

$$
\psi: \operatorname{st}_{\text {Aut } T}(1) \rightarrow \operatorname{Aut} T \times \cdots \times \operatorname{Aut} T
$$

( $d$ сомножителей в произведении), которое сопоставляет элементу $g \in \operatorname{st}_{\text {Aut } T}(1)$ набор $\left(g_{1}, \ldots, g_{d}\right)$, где $g_{i} \in \operatorname{Aut} T, i=1, \ldots, d$, и $g_{i}$ есть сужение автоморфизма $g$ на $i$-е поддерево, растущее из вершины первого уровня.

ОПредЕЛЕниЕ 2. Действие групшы $G$ на регулярном дереве $T$ автоморфизмами называется самоподобным, если сужение стабилизатора $\operatorname{st}_{G}(u)$ на поддерево $T_{u}$ (где $u-$ произвольная вершина) есть група, совпадающая с $G$ при отождествлении $T$ с $T_{u}$.

Условимся под включением $K \succcurlyeq K \times \cdots \times K(d$ сомножителей $)$ понимать включение $\psi(K) \geqslant K \times \cdots \times K$ (при условии, что $K-$ подгруппа из стабилизатора вершины первого уровня).

ОПРЕДЕЛЕНИЕ 3. Группа $G$ называется регулярно ветвящейся, если существует точное сферически транзитивное самоподобное действие $G$ на некотором регулярном 
дереве $T_{d}$ и нормальная подгруппа $K \triangleleft G$ конечного индекса, для которой имеет место включение $K \succcurlyeq K \times \cdots \times K(d$ сомножителей $)$.

В проконечном случае в этом определении надо потребовать, чтобы $G$ была замкнутой подгруппой в Aut $T_{d}$, a $K$ была открытой нормальной подгруппой.

Очевидно, что регулярно ветвящаяся группа является ветвящейся, так как для любого $n$ имеют место включения:

$$
K \times \cdots \times K \preccurlyeq \operatorname{st}_{G}(n) \preccurlyeq G \times \cdots \times G
$$

( $d^{n}$ сомножителей в каждом произведении, смысл второго включения $\preccurlyeq$ аналогичен смыслу первого).

Приведем примеры регулярно ветвящихся грушп. С этой целью для любого натурального числа $p \geqslant 2$ определим группу $G_{p}$, действующую на дереве $T_{p}$.

При $p=2$ группа $G_{2}$ порождена автоморфизмами $a, b, c, d$, где $a$ переставляет два поддерева, растущие из вершин первого уровня, а автоморфизмы $b, c, d$ принадлежат стабилизатору первого уровня и определяются совместными рекурентными соотношениями: $\psi(b)=(a, c), \psi(c)=(a, d), \psi(d)=(1, b)$.

При $p \geqslant 3$ групша $G_{p}$ порождена двумя автоморфизмами $a, t$ (порядка $p$ ) дерева $T_{p}$, где $a$ - циклически переставляет поддеревья, растущие из вершин первого уровня, a $t$ стабилизирует первый уровень и определяется рекурентным соотношением $\psi(t)=(a, 1$, $\ldots, 1, t)$.

ТЕОрема 1. а) Для любого $p \geqslant 2$ группа $G_{p}$ является регулярно ветвящейся группой, причем $K=\gamma_{3}\left(G_{p}\right)$ в случае $p=2$ и $K=G_{p}^{\prime}$ в случае $p \geqslant 3$.

б) Замыкание $\bar{G}_{p}$ әруппы $G_{p}$ в Aut $T_{p}, p \geqslant 2$, является регулярно ветвящейся проконечной группой. Если р является простым числом, то $\bar{G}_{p}$ есть про-р-группа, изоморфная проконечному пополнению $\widehat{G}_{p}$ группы $G_{p}$.

Приведенные примеры являются простейшими примерами ветвящихся групп. Заметим, что группа $G_{2}$ изоморфна группе, исследованной в [3].

3. Связь между ветвящимися и экстремальными группами. Мы назьваем группу әкстремальной, если она бесконечна, но всякая ее истинная фактор-групп конечна. Другие авторы употребляют для этих групा термины “ $\infty$-конечная", "слегка бесконечная" или "едва бесконечная", переводя таким образом на русский язык термин "just infinite", используемьй в англоязычных статьях.

Примерами экстремальных групп являются $\mathbb{Z}, S L(n, \mathbb{Z}) /$ centre, $n \geqslant 3$, а также группы $G_{p}$, построенные вьше (это вытекает из сформулированного ниже критерия экстремальности ветвящихся групш).

Значение класса экстремальных групп в значительной степени определяется следующим утверждением.

ПРЕДЛОЖЕНИЕ 1. а) Всякая бесконечная конечно порожденная группа может быть гомеоморфно отображена на әкстремальную группу.

б) Всякая бесконечная конечно порохсденая проконечная группа, содер жащая про-р-группу ( $p$ - простое число) конечного индекса мохет быть отобрахсена на әкстремальную әруппу.

Заметим, что на произвольные конечно порожденные проконечные групшы утверждение б) не распространяется. 
Из сформулированного предложения следует, что любой вопрос о существовании конечно порожденной группы, обладающей некоторьп свойством, сохраняемым при гомоморфизмах, редуцируется к аналогичному вопросу в классе экстремальных групш.

ОПРЕДЕЛЕниЕ 4. а) Группа назьвается наследственно әкстремальной, если она финитно аппроксимируема и всякая подгруппа конечного индекса в ней является экстремальной.

б) Проконечная группа наследственно әкстремальна, если всякая ее подгруппа конечного индекса является экстремальной.

Например, групшы $\mathbb{Z}, S L(n, \mathbb{Z}) /$ centre, $n \geqslant 3$, являются наследственно экстремальными.

Класс экстремальных груп естественным образом распадается на три непересекающихся подкласса.

Теорема 2. а) Пусть $G$ - экстремальная группа. Тогда либо $G$ является ветвящейся группой, либо $G$ содерэит нормальную подгруппу конечного индекса, разложимую в прямое произведение одного или более экземпляров некоторой груп$n$ пь $L$, причем либо $L$ является наследственно әкстремальной группой, либо $L$ простая аруппа.

б) Пусть $G$ является проконечной әкстремальной группой. Тогда либо $G$ ветвящаяся группа, либо $G$ содержит открытую нормальную подгруппу, разложимую в произведение одного или более әкземпляров некоторой группы L, причем $L$ является наследственно әкстремальной әруппой.

Это утверждение родственно теореме Уилсона, разбивающей класс экстремальных групп на подклассы в зависимости от мощности структурной решетки, связанной с субнормальными подгруппами [4]. Оказывается, что класс групп с субнормальной бесконечной структурной решеткой совпадает с классом ветвящихся групп (см. [1], [5]).

4. Специальные группы. Условимся называть раскраской дерева $T=T_{\bar{m}}$ сопоставление каждой вершине $u$ элемента симметрической групшы $\Sigma_{m_{n}}$, где $n=|u|$ - уровень вершины $u$. Легко видеть, что любой автоморфизм $g \in \operatorname{Aut} T$ однозначно определяется некоторой раскраской $P(g)$ и наоборот. Будем назьвать $P(g)$ портретом элемента $g$, а носителем портрета $P(g)$, обозначаемым supp $P(g)$, назовем множество вершин, раскрашенных неединичным элементом.

Автоморфизм $g$ назьвается корневым, если $\operatorname{supp} P(g)$ совпадает с корневой вершиной. Автоморфизм $g$ финитарен, если $\operatorname{supp} P(g)$ является конечным множеством.

Наконец, назовем $g$ направленным автоморфизмом с направляющим путем $e \in \partial T$, если $g \in \operatorname{stAut}_{T}(e), g$ не является финитарным и любая вершина из множества $\operatorname{supp} P(g)$ удалена от е на расстояние 1.

Так, например, порождающий элемент $а$ группы $G_{p}, p \geqslant 2$, является корневым, а порождающие $b, c, d$ группы $G_{2}$ и порождающий $t$ группы $G_{p}, p>2$, являются направленными с самым крайним справа в дереве $T$ направляющим путем.

Так как выбор направляющего пути не играет существенной роли, то всегда в дальнейшем будем считать, что это самьй крайний правый путь.

Пусть $s$ - направленный автоморфизм. Обозначим через $\pi_{n}(s)$ подгруппу в $\Sigma_{m_{n}}$, порожденную метками портретов $P(s)$ вершин $n$-го уровня. Соответственно если $B<$ Aut $T$ - конечная група, у которой любой неединичньй элемент является направленным автоморфизмом с направляющим путем $e$, то через $\pi_{n}(B)$ обозначим подгруппу в $\Sigma_{m_{n}}$, порожденную группами $\pi_{n}(s), s \in B$. 
Пусть теперь $A$ и $B$ - конечные подгрупшы в Aut $T$, причем $A$ действует транзитивно на первом уровне дерева $T$ и состоит из корневых автоморфизмов, а $B$ состоит из направленных автоморфизмов с направляющим путем $e$ (за исключением единичного элемента), и кроме того, для любого $n=2,3, \ldots$ подгруппа $\pi_{n}(B) \leqslant \Sigma_{m_{n}}$ транзитивна на множестве из $m_{n}$ элементов.

ОПРЕДЕЛЕНИЕ 5. Специальной назьвается любая подгруппа $G \leqslant \operatorname{Aut} T$, порожденная подгруппами $A$ и $B$, удовлетворяющая сформулированньм вьше условиям.

Специальные групш бесконечны. Конструкция специальных груп обладает большими ресурсами. Все известные к настоящему моменту примеры ветвяшихся групп, в том числепостроенные в [1], [6], [7], [8], редуцируются к специальньм. Групшы $G_{p}$ также являются специальными.

Истоки конструкции специальных групп находятся в работах [9], [10], однаков чистом виде эта конструкция впервые была использована в [3] и [6]. В [11] впервые предложено применять указанную конструкцию на деревьях с непостоянньм числом ветвления на различных уровнях.

5. Конгруэнц-свойство и критерий экстремальности. Всякая группа $G$, действующая на дереве $T=T_{\bar{m}}$, обладает естественной фильтрацией $\left\{\operatorname{st}_{G}(n)\right\}_{n=1}^{\infty}$ нормальных подгрупп конечного индекса.

ОПРЕДЕЛЕниЕ 6 . Группа $G$, действующая на дереве, обладает конгруәни-свойством, если всякая подгруппа конечного индекса в $G$ содержит $\operatorname{st}_{G}(n)$ для некоторого $n \geqslant 1$.

Это определение относится к классу абстрактных групп. Если $G$ обладает конгруэнщ-свойством, то замыкание $\bar{G}$ этой групш изоморфно проконечному пополнению $\widehat{G}$. При этом если факторы $G / \operatorname{st}_{G}(n), n=1,2, \ldots$, являются конечными $p$-групшами, то $\widehat{G}$ - про- $p$-групша. Более того, если $G$ является ветвящейся групой, то $\widehat{G}-$ ветвящаяся про- $p$-группа.

Для регулярно ветвяшихся групш существует простой критерий того, когда вьполнено конгруэнц-свойство.

ПРЕДЛОЖЕНИЕ 2. Пусть $G$ - регулярно ветвящаяся группа $u K$ - coответствующая подгруппа из определения 3.

Тогда для $G$ имеет место конгруәни-свойство в том и только том случае, если $K$ содержит $\operatorname{st}_{G}(m)$ при некотором $m \geqslant 1$.

Этот критерий применим ко всем известным регулярно ветвящимся группам, например, к $G_{p}, p \geqslant 2$, группам, построенным в [6], [7], [8], и во всех упомянутых группах конгруэнц-свойство вьполнено.

Существует также простой критерий экстремальности ветвящихся групп. Для группы, действующей на дереве сферически транзитивно, обозначим через $L_{n}$ групш, изоморфную жесткому стабилизатору вершины $n$-го уровня.

ТЕОрема 3. Пусть $G$ - ветвящаяся группа. Тогда $G$ является әкстремальной в том и только том случае, если $\left|L_{n}: L_{n}^{\prime}\right|<\infty, n=1,2, \ldots$.

Этот критерий особенно легко применим в ситуации, когда $G$ действует на регулярном дереве, а последовательность $\left\{L_{n}\right\}_{n=1}^{\infty}$ является периодической или даже постоянной, начиная с некоторого места. Это выполнено для многих регулярно ветвящихся 
групп, в частности, для груп $G_{p}, p \geqslant 2$. Отсюда вытекает, что пополнение $\widehat{G}_{p}, p-$ простое число, является экстремальной конечно порожденной регулярно ветвящейся про- $p$-группой.

Примеры конечно порожденных ветвяшихся периодических $p$-групп, где $p$ - простое число, содержатся в работах [3], [6], [7], [8], [12]. Примеры свободных от кручения ветвящихся групп приведены в [13].

Пополнение ветвящейся периодической групшы, обладающей конгруэнщ-свойством, является ветвящейся про-р-группой со свойством универсальности по вложению (т.е. всякая про- $p$-группа со счетной базой открытых множеств вложима в нее [1], [14]).

ТЕОремА 4. Для любого простого числа р существует конечно порохсенная экстремальная ветвящаяся про-р-группа, свободная от кручения.

Неизвестно, существуют ли примеры групп, удовлетворяющих свойствам, перечисленным в теореме, и не содержаших свободную про- $p$-группу ранга 2 .

6. Максимальные и слабо максимальные подгруппы. Напомним, что подгруппа $H<G$ назьвается максимальной, если всякая подгруппа $M$ такая, что $H \supsetneqq$ $M<G$, совпадает с $G$. Подгруппа $W<G$ бесконечного индекса назьвается слабо максимальной, если любая подгруппа, удовлетворяющая цепочке включений $H \supsetneqq W<G$, имеет конечньй индекс в $G$.

Если $G$ - проконечная группа, то в этом определении надо потребовать, чтобы $W$ была замкнутой подгруппой.

Хорошо известно, что всякая конечно порожденная групша обладает максимальной подгруппой. Однако, существуют конечно порожденные бесконечные группы, все максимальные подгруппы которых имеют конечньй порядок. В то же время, имеет место следующее утверждение.

ПРЕДЛОЖЕНИЕ 3. Пусть $G$ - конечно порожденная бесконечная группа. Тогда в $G$ найдется хотя бы одна слабо максимальная подгруппа.

Для конечно порож денных проконечньх груп, содержащих про- $p$-группу конечного индекса, этот факт отмечен в [15].

Согласно результату Маргулиса и Сойфера [16] группы $S L(n, \mathbb{Z}), n \geqslant 2$, обладают максимальными подгруппами бесконечного индекса. Поэтому и наследственно экстремальные групшы типа $S L(n, \mathbb{Z}) /$ centre, $n \geqslant 3$, также обладают такими подгруппами.

Вопрос о существовании максимальных подгрупп бесконечного индекса в группах ветвящегося типа (например, в группе $G_{2}$ ) поставлен Хартли и долгое время оставался открытым (этот вопрос также обсуждался в [17]). Недавно отрицательньй ответ на него получен в [18]. Наше утверждение состоит в следующем.

ТеОрема 5. Пусть $G_{\omega}-$ произвольная бесконечная $p$-группа $(p \geqslant 2$ - простое число), построенная в [6], [7]. Тогда в $G_{\omega}$ нет максимальных подгрупп бесконечного индекса.

В свете этого результата становится очевидным, что при изучении ветвящихся груш важная роль принадлежит слабо максимальньм подгруппам.

ТеОрема 6. Пусть $G$-ветвящаяся группа, определенная своим действием на дереве $T_{\bar{m}}, e \in \partial T_{m}-$ точка границы, $P=P_{e}=\operatorname{st}_{G}(e)-$ параболическая подгруппа.

Тогда $P$ является слабо максимальной подгруппой. 
Таким образом, получаем континуальное множество слабо максимальных подгрупп, индексированное точками границы дерева. Заметим, что слабая максимальность параболических подгрупп в группах ветвящегося типа впервые отмечена в [13].

Вопрос. Дать описание слабо максимальных подгрупп ветвящихся групп или хотя бы решить указанньй вопрос для конкретных примеров таких, как, скажем, грушы $G_{p}$, $p \geqslant 2$.

Заметим в заключение, что большая часть сформулированных результатов докладывалась на различных конференциях. Например, теоремы 2 и 3 обсуждались на конференции по теории групп в Базе (Англия) в 1997 г. и в Москве на Международной алгебраической конференции памяти А. Г. Куроша [2].

\section{СПИСОК ЦИТИРОВАННОЙ ЛИТЕРАТУРЫ}

[1] Grigorchuk R. I. Just infinite branch groups // New horizons in pro-p-groups. Ed. M. du Sautoy, D. Segal, A. Shalev. Basel: Birkhauser, 2000.

[2] Григорчук Р.И. Об экстремальных и ветвящихся группах // Тезисы докладов международной алгебраической конференции памяти А. Г. Куроша. Москва, 1998. С. 163-165.

[3] Григорчук Р.И. К проблеме Бернсайда о периодических группах // Функцион. анализ и прилож. 1980. Т. 14. №1. С. 53-54.

[4] Wilson J.S. Groups with every proper quotient finite // Proc. Camb. Phil. Soc. 1971. V. 69. P. 373-391.

[5] Wilson J.S. On just infinite abstract and profinite groups // New horizons in pro-p-groups. Ed. M. du Sautoy, D. Segal, A. Shalev. Basel: Birkhauser, 2000.

[6] Григорчук Р.И. Степени роста конечно порожденных групп и теория инвариантньх средних // Изв. АН СССР. Сер. матем. 1984. Т. 48. № 5. С. 939-985.

[7] Григорчук Р. И. О степенях роста $p$-групп и групп без кручения // Матем. сб. 1985. T. 126 . № 2. C. 194-214.

[8] Gupta N., Sidki S. On the Burnside problem for periodic groups // Math. Z. 1983. V. 182. P. 385-388.

[9] Алешин С.В. Конечные автоматы и проблема Бернсайда о периодических группах // Матем. заметки. 1972. Т. 11. № 3. С. 319-328.

[10] Сущанский В. И. Периодические $p$-группы подстановок и неограниченная проблема Бернсайда // Докл. АН СССР. 1979. Т. 247. № 3. С. 557-561.

[11] Рожков А.В. К теории групп алешинского типа // Матем. заметки. 1986. Т. 40. № 5. C. $572-589$.

[12] Рожков А. В. Условия конечности в группах автоморфизмов деревьев // Алгебра и логика. 1998. T. 37. № 2. C. 568-605.

[13] Bartholdi L. P., Grigorchuk R. I. On the parabolic subgroups and Hecke algebras related to some fractal groups. Preprint of FIM. Zurich: ETH-Zentrum, 1999.

[14] Grigorchuk R. I., Herfort W. N., Zaleskii P. A. The profinite completion of certain torsion $p$-groups // Proc. of the International Algebraic Conference in Memory of A. G. Kurosh. Moscow, 1998.

[15] Shalev A. Subgroups structure, fradal dimension, and Kac-Moody algebras // Groups and Geometries. Siena Conference, 1996. Ed. Lino di Martino. Birkhäuser, 1998. P. 163-176.

[16] Margulis G. A., Souifer G. A. Maximal subgroups of infinite index in finitely generated linear groups // J. Algebra. 1981. V. 69. № 1. P. 1-23.

[17] Рожков А. В. Централизаторы элементов в одной группе автоморфиизмов деревьев // Изв. РАН. Сер. матем. 1993. Т. 57. №6. С. 82-105.

[18] Первова Е. Л. Всюду плотные подгруппы групп автоморфизмов деревьев (в печати). 